\title{
Hidrofilicidade de Filmes de Amido/Poli(Butileno Adipato Co-Tereftalato) (Pbat) Adicionados de Tween 80 e Óleo de Soja
}

\author{
Renata P. Herrera Brandelero, Maria Victória Grossmann, Fabio Yamashita \\ Departamento de Ciência e Tecnologia de Alimentos, Universidade Estadual de Londrina
}

\begin{abstract}
Resumo: A incorporação de amido ao polímero poli (butilenoadipatoco-tereftalato) (PBAT) através de blendas com alto teor de amido pode ser uma alternativa para obter embalagens biodegradáveis, minimizar custos e o uso de recursos não renováveis. No entanto, a adição de amido aumenta a permeabilidade ao vapor de água (PVA). A incorporação em filmes com amido de substâncias como óleos vegetais e surfactantes pode diminuir a hidrofilicidade, favorecendo as aplicações destes como embalagens. A hidrofilicidade dos filmes elaborados por blendas de amido/PBAT adicionados de óleo de soja (OS) e tween 80 (TW) foi avaliada considerando o efeito do OS e TW nas isotermas de sorção dos filmes, na PVA e nos coeficientes de difusão $\left(D^{w}\right)$ e solubilidade $(\beta)$ do vapor de água. Filmes com OS com ou sem TW apresentaram menor quantidade de água de sorção, sendo os filmes com menores quantidades de OS e sem TW menos hidrofílicos e menos permeáveis aos vapores de água. A adição de OS reduziu os valores de $\beta$ e $\mathrm{D}^{\mathrm{w}}$ dos filmes de amido/PBAT. O efeito foi relacionado com o aumento das porções hidrofóbicas e da compatibilidade entre o amido e PBAT na presença de OS.
\end{abstract}

Palavras-chave: Polímeros biodegradáveis, extrusão de sopro, blendas.

\section{Hydrophilicity of Starch and Poly(Butylene Adipate-Co-Terephthalate) (Pbat) Films Containing Tween 80 and Soybean Oil}

\begin{abstract}
Incorporating starch into the poly(butylene adipate-co-terephthalate) (PBAT) polymer by means of blends with high starch contents is a possible option for producing biodegradable packaging using renewable resources and reducing costs. However, the addition of starch increases the water vapour permeability (WVP). The incorporation of substances as lipids and surfactants can reduce the hydrophilicity of films containing starch, favouring their use as packaging. The hydrophilicity of films produced from blends of starch/PBAT with added soybean oil (SO) and tween 80 (TW) was studied. The effects of these substances on the sorption isotherm, on the WVP and on the water vapour diffusion $\left(\mathrm{D}^{\mathrm{w}}\right)$ and solubility $(\beta)$ coefficients of the films were evaluated. The water sorption in films with SO, with or without TW, was low. The films with the lowest amount of SO and without TW were the least hydrophilic and less permeable to water vapour. Adding SO reduced the $\beta$ and $\mathrm{D}^{\mathrm{w}}$ values of the starch/PBAT films. This effect was related to the increase in the hydrophobic portions and the compatibility between starch and PBAT in the presence of SO.
\end{abstract}

Keywords: Biodegradable polymers, blow extrusion, blends.

\section{Introdução}

O emprego de polímeros degradáveis na fabricação de embalagens como sacolas, sacos de lixos, plásticos para uso na agricultura, embalagens para os serviços de alimentação entre outros produtos de uso rápido pode minimizar o acúmulo dos resíduos plásticos no ambiente ${ }^{[1]}$. No entanto a confecção de filmes degradáveis é limitada pelo custo e pela capacidade de produção da maioria dos polímeros degradáveis (polilactato, polihidroxibutirato, policaprolactona entre outros). Estes limitadores podem ser atenuados pela incorporação biopolímeros, como o amido e proteínas através da elaboração de blendas ${ }^{[2]}$.

$\mathrm{O}$ amido de mandioca é especialmente abundante em países tropicais, comparado ao amido de milho apresenta menor valor tecnológico e menores preços de comercialização, sendo o uso em filmes uma alternativa de valorização desta matéria-prima. O amido apresenta compatibilidade com processos de extrusão utilizados na elaboração de filmes convencionais e na presença de plastificantes dá origem a um material com características termoplásticas, conhecido como amido termoplástico (ATP) ${ }^{[3,4]}$. O poli(butileno adipato co-tereftalato) (PBAT) é um polímero biodegradável que apresenta propriedades mecânicas similares os filmes de polietileno, porém maior permeabilidade ao vapor de água; sendo obtido por síntese a partir de derivados do petróleo ${ }^{[5]}$.
Filmes elaborados a partir de blendas entre polímeros termoplásticos e amido apresentam perdas nas propriedades mecânicas e de barreira ao vapor de água com o aumento do teor de amido na blenda ${ }^{[1-6]}$. Na elaboração de embalagem para alimentos o uso do amido pode favorecer reações entre a embalagem e o alimento e a diminuição da vida de prateleira. $\mathrm{O}$ aumento da permeabilidade ao vapor de água (PVA) e da absorção de água é um dos principais fatores que podem contribuir para a degradação de embalagens de filmes de amido, bem como do alimento embalado.

A adição de substâncias hidrofóbicas pode atuar como barreira ao vapor de água em filmes de amido. Estas sustâncias podem originar regiões na matriz do filme com menor polaridade que o amido, exercendo efeito de barreira através da redução da água adsorvida na superfície do filme ${ }^{[7]}$. A eficiência da substância hidrofóbica como barreira ao vapor de água pode estar relacionada com a razão entre as porções hidrofílicas e hidrofóbicas do filme e com as características da substância adicionada como polaridade, presença de insaturações ou ramificações ${ }^{[8]}$.

Várias pesquisas de biofilmes aditivados com óleos, ácidos graxos e surfatantes foram realizadas ${ }^{[8-13]}$. Alguns estudos apontam melhorias na barreira ao vapor de água com o aumento da cadeia carbônica da substância hidrofóbica e quando uma razão específica entre as porções hidrofílicas e hidrofóbicas do filme é estabelecida ${ }^{[10,14]}$.

Autor para correspondência: Renata P. Herrera Brandelero, Departamento de Ciência e Tecnologia de Alimentos, Universidade Estadual de Londrina - UEL, Rod. Celso Garcia Cid (PR 445), Km 380, CP 6001, CEP 86051-990, Londrina, PR, Brasil, e-mail: renataherrera@yahoo.com.br 
O óleo de soja é uma matéria-prima disponível e barata quando comparada aos ácidos graxos e apresenta ponto de fusão compatível com processos de extrusão. $\mathrm{O}$ uso de surfatante como o monooleato de sorbitana, conhecido com o nome comercial de tween 80 (TW), também é estudado como forma de diminuir a higroscopicidade dos filmes de amido. No entanto alguns pesquisadores verificaram aumento da PVA e redução na resistência mecânica com a incorporação de óleos, seja pelo aumento das zonas amorfas no filme ou pela ocorrência de separação de fases o que ocasiona prejuízos na microestrutura do filmes, favorecendo o processo de difusão do vapor de água ${ }^{[11]}$. Assim a incorporação de surfatantes associados ao óleo poderia reduzir os prejuízos à estrutura dos filmes ${ }^{[8,15]}$.

Os surfatantes seriam mais eficazes que óleos devido à natureza anfifílica da molécula, pois a presença de regiões hidrofílicas aumenta a compatibilidade com o amido, diminuindo a ocorrência de separação de fases e a associação da porção hidrofílica do surfatante com o amido pode reduzir os sítios de ligação para água ${ }^{[14]}$.

A PVA de filmes que contém amido é mais complexa que de filmes elaborados somente com resinas plásticas convencionais, pois além dos processos difusivos ocorre a interação do amido com a água e mecanismos de sorção influenciam a permeabilidade ${ }^{[16]}$. Muller, Yamashita e Laurindo ${ }^{[17]}$ estimaram a partir dos parâmetros dos modelos de GAB e BET, que modelam as isotermas de sorção de água, o coeficiente de solubilidade e de difusão, sendo a higroscopicidade avaliada através da definição destes coeficientes. Verificaram que o aumento da solubilidade da água na matriz do filme é o principal fator para o aumento da PVA e que este coeficiente é influenciado tanto pela formulação do filme (concentração e tipo de plastificante) como pela umidade ambiente.

Poucos trabalhos avaliaram a adição de substâncias hidrofóbicas em filmes elaborados por blendas de amido com outro polímero, como também são escassos os trabalhos que avaliaram a adição destas substâncias em filmes de amido elaborados por extrusão. O objetivo do trabalho foi avaliar o efeito da adição de óleo de soja (OS) e tween 80 (TW) na higroscopicidade de filmes elaborados por blendas de amido/PBAT através da estimativa dos coeficientes de solubilidade e difusão em diferentes condições de umidade.

\section{Material e Métodos}

\section{Material}

$\mathrm{O}$ amido de mandioca (Manihot esculenta) foi fornecido pela Indemil (Diadema SP), sob marca AmidoMani. O polímero biodegradável poli(butileno adipato co-tereftalato) (PBAT) foi fornecido pela empresa BASF sob nome comercial de Ecoflex ${ }^{\circledR}$-F. O glicerol comercial (Dinâmica, Brasil) foi utilizado como plastificante e o surfatante utilizado foi o monooleato de sorbitan etoxilado (Synth, Brasil), nome comercial tween 80 (TW), com balanço hidrofílico-lipofílico (HLB) igual a 15,0 e densidade de
$1,07 \mathrm{~g} . \mathrm{cm}^{-3}$. O óleo de soja refinado (OS) procedente da refinadora Bunge Alimentos S.A foi adquirido no mercado local.

\section{Elaboração das blendas e dos filmes}

Os filmes foram processados em extrusora piloto mono-rosca marca BGM (modelo EL-25, Brasil) por sistema de sopro em balão, equipada com rosca de $250 \mathrm{~mm}$ de diâmetro, matriz circular com anel de resfriamento para formação de filmes com 150 a $300 \mathrm{~mm}$ de diâmetro, bobinador e granulador. A velocidade da rosca foi mantida em $30 \mathrm{rpm}$, o programa de temperatura utilizado foi de $120^{\circ} \mathrm{C}$ para as 3 zonas do canhão, $120^{\circ} \mathrm{C}$ para a matriz formadora dos pellets e $125^{\circ} \mathrm{C}$ na zona 1 e $130{ }^{\circ} \mathrm{C}$ na zona 2 da matriz circular do balão.

Foram processados filmes de amido/PBAT com concentrações de $65 \mathrm{~g}$ amido/100 g amido+PBAT adicionados de glicerol, óleo de soja e tween 80 nas quantidades descritas na Tabela 1 . Filmes com $100 \%$ de amido (F100) e com 65\% de amido (F0) sem surfatante e/ou óleo de soja foram elaborados. Os filmes F100 e F0 foram adicionados de $30 \%$ de glicerol sob massa de amido, já em filmes com OS e/ou TW a quantidade de glicerol teve que ser diminuída para melhorar o fluxo do material termoplástico na direção da matriz de saída.

O amido, glicerol, tween 80 e óleo de soja foram homogeneizados e extrudados para produção de pellets de amido termoplástico (ATP). Os pellets de ATP foram misturados aos pellets de PBAT e extrudados, produzindo pellets de ATP+PBAT que foram extrudados novamente para obter um material mais homogêneo. Os filmes foram produzidos pelo sistema de sopro em balão, bobinados e armazenados em embalagens de papel laminado até o momento das análises. Os filmes de amido foram produzidos a partir da extrusão do amido e do glicerol, ao total foram realizadas três extrusões para padronizar o cisalhamento sofrido pelo amido.

\section{Determinação das isotermas de sorção}

As isotermas de sorção dos filmes foram determinadas em diferentes umidades relativas (UR) $(11,8 \%, 32,8 \%, 43,2 \%, 52,9 \%$, 64,5 e $90 \%$ ) a $25{ }^{\circ} \mathrm{C}$, conforme Mali et al. ${ }^{[18]}$. Corpos de provas $(25 \mathrm{~mm} \times 25 \mathrm{~mm}$ ) foram condicionados por 30 dias em cloreto de cálcio $\left(\mathrm{CaCl}_{2}\right)$ com a finalidade de diminuir a umidade dos filmes para valores menores que $11,8 \%$ UR. Posteriormente foram colocados em recipientes fechados contendo soluções salinas saturadas para obter a UR desejada. Os filmes foram pesados em intervalos regulares até obter três pesagens consecutivas iguais (condição de equilíbrio). A umidade absoluta (em base seca) foi determinada pelo método em estufa $\left(105^{\circ} \mathrm{C}, 24\right.$ horas). Todos os testes foram conduzidos em triplicata. As isotermas foram modeladas a partir dos valores de umidades obtidos em $11,8 \%$ de UR utilizando o modelo de Guggenhein-Anderson-de Bôer (GAB) conforme Equação 1. O programa Statistica 6.0 foi utilizado para realizar a modelagem. Os parâmetros convergiram pelo método de Quasi-Newton.

Tabela 1. Composição das formulações dos filmes elaborados a partir de blendas de ATP/PBAT adicionadas de óleo de soja e/ou tween 80.

\begin{tabular}{|c|c|c|c|c|c|}
\hline Formulação & $\begin{array}{c}\text { Amido } \\
(\mathrm{g} / 100 \mathrm{~g} \text { amido+PBAT })\end{array}$ & $\begin{array}{c}\text { PBAT } \\
(\mathrm{g} / 100 \mathrm{~g} \text { amido+PBAT })\end{array}$ & $\begin{array}{c}\text { Glicerol } \\
\text { (g/100 g amido) }\end{array}$ & $\begin{array}{c}\text { Óleo } \\
\text { (g/100 g amido) }\end{array}$ & $\begin{array}{c}\text { Tween } \\
\text { (g/100 g amido) }\end{array}$ \\
\hline F0 & 65 & 35 & 30 & 0 & 0 \\
\hline $\mathrm{F} 1$ & 65 & 35 & 24,5 & 0,5 & 0 \\
\hline $\mathrm{F} 2$ & 65 & 35 & 24,0 & 1,0 & 0 \\
\hline F3 & 65 & 35 & 24,0 & 0,5 & 0,5 \\
\hline $\mathrm{F} 4$ & 65 & 35 & 23,5 & 1,0 & 0,5 \\
\hline F5 & 65 & 35 & 23,5 & 0,5 & 1,0 \\
\hline F6 & 65 & 35 & 23 & 1,0 & 1,0 \\
\hline F100 & 100 & 0 & 30 & 0 & 0 \\
\hline
\end{tabular}




$$
X_{w} \frac{C \cdot k \cdot m_{0} \cdot a_{w}}{\left[\left(1-k \cdot a_{w}\right)\left(1-k \cdot a_{w}+C \cdot k \cdot a_{w}\right)\right]}
$$

onde: C (calor de sorção da monocamada), k (calor de sorção da multicamada) e $\mathrm{m}_{\mathrm{o}}$ (monocamada dada em g água/g sólidos) são os parâmetros do modelo, $\mathrm{X}_{\mathrm{w}}$ é a umidade em base seca (g água/g sólidos) e $\mathrm{a}_{\mathrm{w}}$ é a UR/100.

\section{Determinação da PVA}

Na determinação da permeabilidade dos filmes foi utilizado o método gravimétrico adaptado da normativa E 96-95 da ASTM ${ }^{[19]}$. A PVA foi determinada mantendo gradiente de umidade relativa (UR) próxima a $30 \%$ porém, optou-se por variar os valores absolutos das UR. Os intervalos de UR utilizados foram iguais a $(2 \%-32,8 \%),(32,8 \%-64,5 \%)$ e $(64,5-90 \%)$. Os corpos de prova foram condicionados a $64,5 \%$ UR a $25^{\circ} \mathrm{C}$ por 48 horas e colocados em cápsulas parcialmente preenchidas com $\mathrm{CaCl}_{2}$ (2\% UR), soluções saturadas de cloreto de magnésio $\left(\mathrm{MgCl}_{2}\right)(32,8 \%)$ e nitrato de sódio $\left(\mathrm{NaNO}_{2}\right)(64,5 \%)$. Os corpos de prova foram fixados na abertura circular da cápsula e seladas com graxa de silicone. As cápsulas foram acondicionadas em dessecador nas condições de UR de $32,8 \%, 64,5 \%$ e $90 \%$ a $25{ }^{\circ} \mathrm{C}$. A pesagem das cápsulas foi realizada em intervalos de 12 horas por 5 dias consecutivos. A PVA foi calculada em g.m 1 .dia-1.Pa ${ }^{-1}$ utilizando a Equação 2. A determinação da permeabilidade foi realizada em duplicata.

$$
\mathrm{PVA}=\frac{\mathrm{TPVA} \cdot \delta}{\mathrm{A} \cdot \mathrm{P}_{\mathrm{S}} \cdot\left(\frac{U R_{1}-U R_{2}}{100}\right)}
$$

onde: TPVA é a taxa de permeabilidade ao vapor de água (g água/dia), $\delta$ é a espessura média dos filmes $(\mathrm{m})$, A é a área de permeação da cápsula $\left(28,26 \times 10^{-4} \mathrm{~m}^{2}\right)$, $\mathrm{P}_{\mathrm{s}}$ é a pressão de saturação do vapor de água a $25^{\circ} \mathrm{C}(163.678,8 \mathrm{~Pa})$ e a UR é a umidade relativa no interior do dessecador (sub-índice 1) e no interior da cápsula (sub-índice 2).

\section{Determinação da densidade e espessura}

A espessura dos filmes foi determinada utilizando-se um micrômetro manual (resolução 0,001 mm, Mitutoyo, Brasil). A espessura final foi determinada pela média aritmética de 30 medidas em 6 corpos de prova condicionados por 48 horas a $64 \%$ UR, com 5 medidas aleatórias em cada corpo de prova. A densidade foi determinada em corpos de prova cortados em quadrados de $25 \times 25 \mathrm{~mm}$, condicionadas por 30 dias em dessecador com cloreto de cálcio, após este período foram pesados para o cálculo da densidade, sendo esta a média aritmética de 18 determinações.

\section{Determinação dos coeficientes de solubilidade ( $\beta$ ) e de difusão ( $\left.D^{w}\right)$}

A metodologia utilizada para o cálculo do coeficiente de solubilidade $(\beta)$ foi proposta por Larotonda et al. ${ }^{[20]}$ a partir da derivada de primeira ordem do modelo de GAB, que correlaciona a umidade em função da atividade de água dividida pela pressão de vapor de água $\left(\mathrm{p}_{\mathrm{s}}\right.$ ) na temperatura de $25^{\circ} \mathrm{C}$, conforme a Equação 3.

$$
\beta=\frac{C k m_{o}}{p_{s}}\left[\begin{array}{l}
\frac{1}{\left(1-k a_{w}\right)\left(1-k a_{w}+C k a_{w}\right)}- \\
{\left[\begin{array}{l}
a_{w} \\
{\left[-k\left(1-k a_{w}\right)\left(1-k a_{w}+C k a_{w}\right)\right]^{2}}
\end{array}\right.} \\
\left.\left[-C k a_{w}\right)+\left(1-k a_{w}\right)(-k+C k)\right]
\end{array}\right]
$$

onde: $\beta$ é o coeficiente de solubilidade e $\mathrm{C}, \mathrm{k}$ e $\mathrm{m}_{\mathrm{o}}$ são os parâmetros do modelo de $\mathrm{GAB}, \mathrm{p}_{\mathrm{s}}$ é a pressão de vapor de água a $25^{\circ} \mathrm{C}$. O valor de $\mathrm{a}_{\mathrm{w}}$ utilizado foi a média do gradiente de UR/100 descrito no item 2.4.

$\mathrm{O}$ coeficiente de difusão do vapor de água $\left(\mathrm{D}^{\mathrm{w}}\right)$ foi determinado a partir dos valores de $\beta$ e dos valores de PVA determinadas para os filmes nas condições de umidade do item 2.4 , através da equação 4 , sendo $\rho\left(\mathrm{g} \cdot \mathrm{cm}^{-3}\right)$ a densidade dos filmes.

$$
D^{w}=\frac{P V A}{\rho \cdot \beta}
$$

\section{Resultados e Discussão}

Os filmes apresentaram expansão para formação do balão e foram bobináveis, apresentando boa processabilidade. As formulações com adição de óleo de soja e/ou tween 80 resultaram em pellets pegajosos quando adicionados de $30 \%$ de glicerol e esta quantidade foi reduzida para $25 \%$ (com relação à massa de amido) (Tabela 1), já filmes com 65\% (F0) ou 100\% (F100) de amido sem adição de OS e/ou TW apresentaram melhor processabilidade com 30\% de glicerol. Filmes F100 e F0 foram considerados bons referenciais para as demais formulações (F1 a F6) uma vez que foram obtidos sob as mesmas condições de processo e mantidos na discussão dos resultados.

\section{Isotermas de sorção}

As isotermas dos filmes estão apresentadas na Figura 1. Os parâmetros obtidos através da modelagem das isotermas usando o modelo de GAB estão apresentados na Tabela 2. O ajuste do modelo aos dados foi considerado satisfatório e similar aos obtidos por outros pesquisadores da área de biofilmes ${ }^{[17,19-22]}$. $\mathrm{O}$ parâmetro $\mathrm{C}$ dos filmes obtidos por blendas entre o amido e PBAT não foi significativo, enquanto os parâmetros $\mathrm{k}$ e $\mathrm{m}$ foram significativos $(\mathrm{p}<0,05)$.

Comparando os valores de $m_{0}$ e da água de sorção (Tabela 2) dos filmes adicionados de OS (F1 a F6) com os dos filmes de amido (F100) e com os filmes obtidos por blendas de amido/PBAT sem aditivos (F0) verifica-se que estes foram bem menores, indicando que o óleo exerceu efeito de barreira ao vapor de água e diminuiu a hidrofilicidade dos filmes.

Costa $^{[22]}$ obteve para filme produzido a partir de blenda de amido termoplástico: PBAT (70:30) um valor de $\mathrm{m}_{0}$ igual a 0,073 g água/g sólidos e água de sorção de $0,70 \mathrm{~g}$ água/g sólidos, sendo que o ATP continha $20 \%$ de glicerol. Já Müller et al. ${ }^{[17]}$, para filme elaborado com $100 \%$ de ATP contendo 30\% de glicerol, obtiveram valor de $\mathrm{m}_{0}$ igual a 0,094 g água/g sólidos (ambos a 90\% UR). Estes valores foram superiores aos determinados para filmes adicionados de OS e similares aos filmes de amido ou amido/PBAT elaborados neste trabalho, indicando que nos filmes de amido/PBAT com OS a redução

Tabela 2. Parâmetros do modelo de GAB para as isotermas de sorção de filmes de amido/PBAT adicionados de óleo de soja e tween 80 .

\begin{tabular}{ccccc}
\hline \multirow{2}{*}{ Formulação } & \multicolumn{4}{c}{ Parâmetros do modelo de GAB } \\
\cline { 2 - 5 } & $\mathbf{C}^{*}$ & $\mathbf{k}$ & $\mathbf{m}_{\mathbf{0}}$ & $\mathbf{R}^{\mathbf{2}}$ \\
\hline F0 & 212 & 0,90 & 0,112 & 0,98 \\
F1 & 133 & 0,91 & 0,067 & 0,98 \\
F2 & 215 & 0,94 & 0,062 & 0,99 \\
F3 & 60 & 0,94 & 0,070 & 0,97 \\
F4 & 403 & 0,92 & 0,080 & 0,99 \\
F5 & 186 & 0,93 & 0,066 & 0,97 \\
F6 & 214 & 0,92 & 0,062 & 0,99 \\
F100 & 7 & 0,97 & 0,090 & 0,99 \\
\hline
\end{tabular}

*parâmetros C não foram significativos ao nível de 0,05 . 


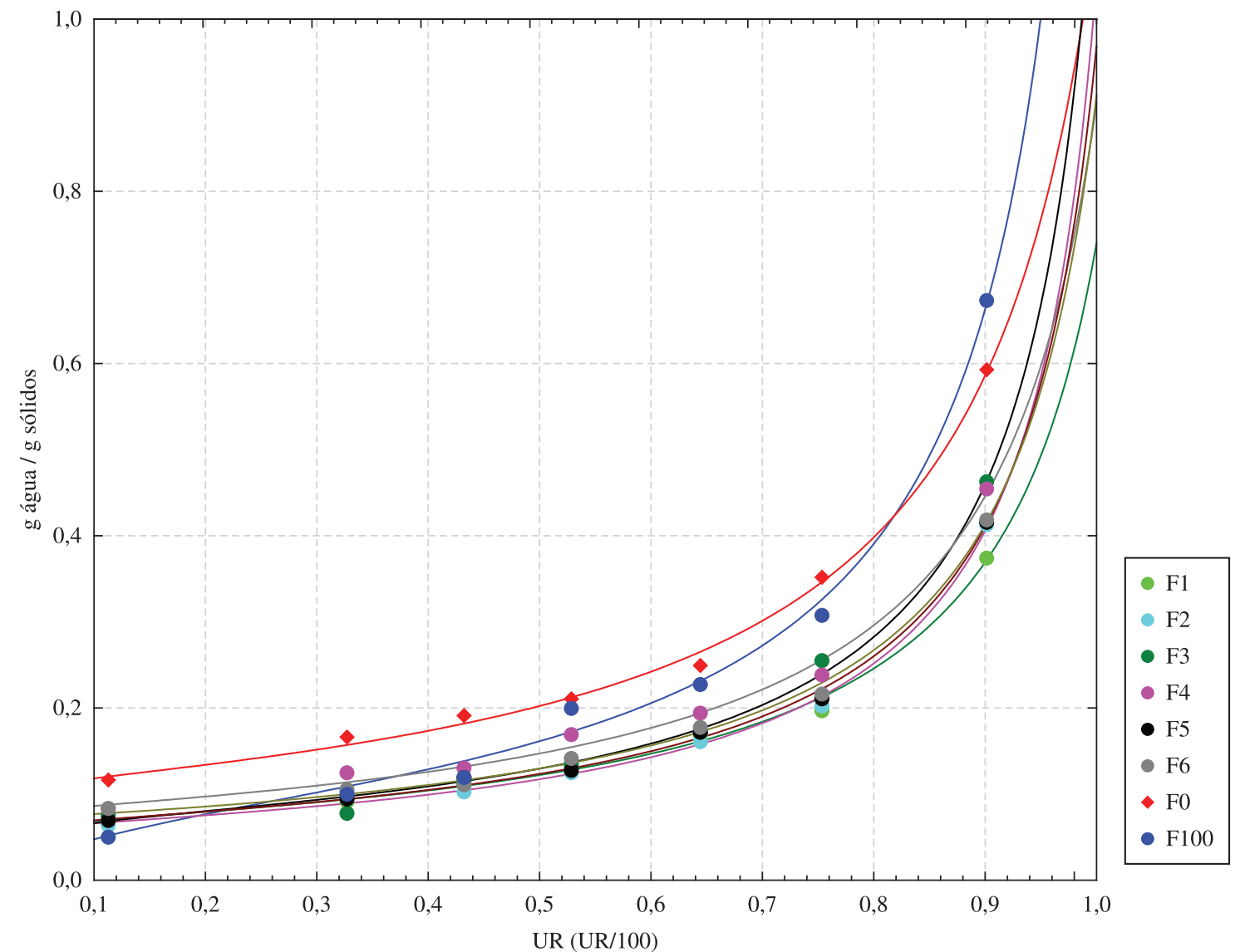

Figura 1. Isotermas de sorção obtidas para filmes de amido/PBAT (F0), filmes adicionados óleo de soja (F1 e F2) e filmes adicionados de tween 80 e óleo de soja (F3-F6) e filmes apenas de amido (F100).

da higroscopicidade foi maior que nos filmes de amido/PBAT sem OS com mesma concentração de PBAT, confirmando que a redução da higroscopicidade ocorreu devido ao OS.

Filmes adicionados de $0,5 \%$ de TW (F3 e F4) apresentaram os maiores valores de água de sorção na monocamada $\left(\mathrm{m}_{0}\right)$ e foram os mais hidrofílicos quando comparados aos demais filmes (Figura 1). $\mathrm{O}$ efeito, provavelmente, se deve ao aumento da disponibilidade de sítios de ligação para água pela presença da parte hidrofílica do surfatante o que aumenta a água de sorção. Com adição de $1 \%$ de surfatante (F5 e F6) os filmes apresentaram valores de $m_{0}$ menores que os com $0,5 \%$ de TW (F3 e F4) e similares aos dos filmes F1 e F2 (sem adição de surfatante e óleo). Este comportamento ocorreu, provavelmente, pelo aumento de porções hidrofóbicas nos filmes devido ao aumento da concentração do surfatante o que contrabalanceou a ação da parte hidrofílica do mesmo.

O parâmetro k (Tabela 2) indica a energia de sorção do vapor de água entre as multicamadas, quando $\mathrm{k}=1$ admite-se que não há interações do vapor de água nas multicamadas ou que não há variação da energia de sorção nas multicamadas o que ocorre num sólido homogêneo, explicando os maiores valores de $\mathrm{k}$ para filmes com $100 \%$ de amido. Filmes com óleo apresentaram valores de $\mathrm{k}$ superiores aos sem adição de óleo ou surfatante, provavelmente, por uma melhora na microestrutura na presença de OS.

\section{Permeabilidade ao vapor de água, coeficientes de solubilidade ( $\beta$ ) e de difusão $\left(D^{w}\right)$ dos filmes}

Os valores da PVA e dos coeficientes de $\beta$ e $\mathrm{D}^{\mathrm{w}}$ encontrados para os filmes em diferentes condições de umidade estão apresentados na Tabela 3, assim como os valores de densidade $(\rho)$ e espessura.
Em baixa umidade relativa $(2-32,8 \%)$ a permeação do vapor de água foi controlada pelo coeficiente de solubilidade e os valores de PVA foram os mais baixos. Com o aumento da umidade para o intervalo de 33-64\% houve aumento do coeficiente de solubilidade, filmes adicionados com $0,5 \%$ de OS e sem TW foram os mais resistentes a esta variação e apresentaram menor PVA que os demais filmes. No intervalo de 64-90\% UR os filmes apresentaram maior coeficiente de solubilidade do vapor de água na matriz do filme o que elevou a PVA dos filmes analisados.

O aumento da solubilidade do vapor de água na matriz do filme favorece a permeação do vapor de água devido à saturação dos sítios de ligação com a água, diminuindo a energia necessária para sorção ${ }^{[16]}$. Muller et al. ${ }^{[17]}$, Costa ${ }^{[22]}$ e Matta et al. ${ }^{[23]}$ verificaram comportamento semelhante ao deste trabalho para a relação entre a PVA e $\beta$, estabelecendo que a PVA dos filmes de amido é controlada pela solubilidade do vapor de água na matriz do filme, sendo o valor de $\beta$ dependente da umidade ambiente.

A PVA dos filmes de amido/PBAT sem adição de OS e/ou TW (F0) foi igual a $2,41 \times 10^{-7} \mathrm{~g} \cdot \mathrm{dia}^{-1} \cdot \mathrm{Pa}^{-1} \cdot \mathrm{m}^{-1}(32-64 \% \mathrm{UR})$, menor que a PVA de filmes preparados somente com amido (F100) o que se deve pela diminuição dos valores do coeficiente de solubilidade $(\beta)$ (Tabela 3). O efeito está relacionado à redução dos sítios de ligação para água devida à presença de PBAT. No entanto, apesar da redução da PVA na presença de PBAT, filmes de PBAT/amido apresentaram PVA maior que a dos filmes elaborados com resinas convencionais, como os de PEBD $\left(0,8 \times 10^{-7} \mathrm{~g} \cdot \mathrm{dia}^{-1} \cdot \mathrm{Pa}^{-1} \cdot \mathrm{m}^{-1}\right)$, filmes de polilactados $\left(1 \times 10^{-7} \mathrm{~g} \cdot \mathrm{dia}^{-1} \cdot \mathrm{Pa}^{-1} \cdot \mathrm{m}^{-1}\right)$ e filmes de polihidroxibutiratos $\left(0,1 \times 10^{-7}\right.$ g. $\left.\operatorname{dia}^{-1} \cdot \mathrm{Pa}^{-1} \cdot \mathrm{m}^{-1}\right)$ determinados sob gradiente de $0-90 \% \mathrm{UR}^{[24]}$. 
Tabela 3. Permeabilidade ao vapor de água (PVA), espessura (esp), densidade ( $\rho$ ), coeficiente de solubilidade $(\beta)$, coeficiente de difusão $\left(\mathrm{D}^{\mathrm{w}}\right)$ dos filmes de amido/PBAT adicionados de óleo de soja (OS) e tween 80 (TW).

\begin{tabular}{|c|c|c|c|c|c|c|}
\hline Filme & $\begin{array}{c}\Delta \mathbf{U R} \\
(\%)\end{array}$ & $\begin{array}{c}\text { PVA }^{*}\left(\times 10^{7}\right) \\
\left(\text { g.dia }^{-1} \cdot \mathbf{P a}^{-1} \cdot \mathbf{m}^{-1}\right)\end{array}$ & $\begin{array}{l}\text { Esp } \\
(\mu \mathbf{m})\end{array}$ & $\begin{array}{c}\rho \\
\left(\mathrm{g.cm}^{-3}\right)\end{array}$ & $\begin{array}{l}\beta\left(\times 10^{6}\right) \\
(\mathrm{g} / \mathrm{g} \mathrm{Pa})\end{array}$ & $\begin{array}{c}\mathrm{D}^{\mathrm{w}}\left(\times 10^{8}\right) \\
\left(\mathrm{m}^{2} / \mathrm{h}\right)\end{array}$ \\
\hline F0 & $2,0-32,8$ & $0,60^{\mathrm{b}}$ & $200^{\mathrm{b}}$ & 1,34 & 0,80 & 6,00 \\
\hline \multirow{2}{*}{ ( $0 \%$ TW, $0 \%$ OS, $65 \%$ amido) } & $32,8-64,5$ & $2,41^{\mathrm{b}}$ & & & 1,96 & 9,20 \\
\hline & $64,5-90,0$ & $3,11^{\mathrm{b}}$ & & & 7,22 & 3,20 \\
\hline $\mathrm{F} 1$ & $2,0-32,8$ & $0,37^{\mathrm{b}}$ & $221^{\mathrm{b}}$ & 0,91 & 0,63 & 4,45 \\
\hline \multirow{2}{*}{$\begin{array}{c}\text { (0\% TW, } 0,5 \% \text { OS } \\
65 \% \text { amido) }\end{array}$} & $32,8-64,5$ & $0,73^{\mathrm{d}}$ & & & 1,27 & 6,33 \\
\hline & $64,5-90,0$ & $3,09^{\mathrm{b}}$ & & & 4,53 & 2,85 \\
\hline $\mathrm{F} 2$ & $2,0-32,8$ & $0,36^{\mathrm{b}}$ & $237^{\mathrm{b}}$ & 1,26 & 0,57 & 5,30 \\
\hline \multirow{2}{*}{ ( $0 \%$ TW, $1 \%$ OS, $65 \%$ amido) } & $32,8-64,5$ & $1,43^{\mathrm{bc}}$ & & & 1,21 & 9,30 \\
\hline & $64,5-90,0$ & $4,70^{\mathrm{b}}$ & & & 4,61 & 8,10 \\
\hline $\mathrm{F} 3$ & $2,0-32,8$ & $0,40^{\mathrm{b}}$ & $119^{\mathrm{b}}$ & 1,18 & 0,79 & 4,31 \\
\hline \multirow{2}{*}{$\begin{array}{c}(0,5 \% \mathrm{TW}, 0,5 \% \text { OS } \\
65 \% \text { amido })\end{array}$} & $32,8-64,5$ & $1,45^{\text {bc }}$ & & & 1,39 & 8,81 \\
\hline & $64,5-90,0$ & $3,52^{\mathrm{b}}$ & & & 5,40 & 5,53 \\
\hline F4 & $2,0-32,8$ & $0,43^{\mathrm{b}}$ & $145^{\mathrm{b}}$ & 1,20 & 0,70 & 5,37 \\
\hline \multirow{2}{*}{$\begin{array}{c}(0,5 \% \mathrm{TW}, 1,0 \% \text { OS } \\
65 \% \text { amido })\end{array}$} & $32,8-64,5$ & $1,72^{\mathrm{bc}}$ & & & 1,44 & 9,94 \\
\hline & $64,5-90,0$ & $3,56^{\mathrm{b}}$ & & & 5,3 & 5,57 \\
\hline F5 & $2,0-32,8$ & $0,35^{\mathrm{b}}$ & $173^{\mathrm{b}}$ & 1,12 & 0,61 & 5,12 \\
\hline \multirow{2}{*}{$\begin{array}{c}(1 \% \mathrm{TW}, 0,5 \% \mathrm{OS}, \\
65 \% \text { amido })\end{array}$} & $32,8-64,5$ & $1,04^{\mathrm{bc}}$ & & & 1,26 & 7,37 \\
\hline & $64,5-90,0$ & $3,00^{\mathrm{b}}$ & & & 4,80 & 5,61 \\
\hline F6 & $2,0-32,8$ & $0,60^{\mathrm{b}}$ & $118^{\mathrm{b}}$ & 1,56 & 0,57 & 6,12 \\
\hline \multirow{2}{*}{$\begin{array}{c}(1 \% \mathrm{TW}, 1,0 \% \mathrm{OS}, \\
65 \% \text { amido) }\end{array}$} & $32,8-64,5$ & $1,45^{\mathrm{bc}}$ & & & 1,23 & 7,58 \\
\hline & $64,5-90,0$ & $3,14^{\mathrm{b}}$ & & & 4,80 & 4,19 \\
\hline F100 & $2,0-32,8$ & $2,84^{\mathrm{a}}$ & $334^{a}$ & 2,24 & 1,49 & 8,5 \\
\hline \multirow{2}{*}{$\begin{array}{c}(0 \% \mathrm{TW}, 0 \% \mathrm{OS}, \\
100 \% \text { amido })\end{array}$} & $32,8-64,5$ & $7,52^{\mathrm{a}}$ & & & 2,08 & 16,0 \\
\hline & $64,5-90,0$ & $20,51^{\mathrm{a}}$ & & & 8,29 & 11,1 \\
\hline
\end{tabular}

*Letras comparam a PVA dos filmes obtida no mesmo intervalo de umidade, médias com letras iguais não diferem ao nível de 0,05 pelo teste de Tukey.

Filmes com adição de $0,5 \%$ de OS sem adição de surfatante apresentaram PVA, significativamente, menor $(\mathrm{p}<0,05)$ que os demais filmes quando o intervalo de 33-64\% umidade foi utilizado (Tabela 3), sendo a PVA sob esta condição menor que a PVA de filmes de PEBD $\left(0,8 \times 10^{-7} \mathrm{~g} \cdot \mathrm{dia}^{-1} \cdot \mathrm{Pa}^{-1} \cdot \mathrm{m}^{-1}\right)^{[24]}$. Este efeito foi relacionado com a combinação da diminuição dos coeficientes de solubilidade e de difusão do vapor de água, indicando que a presença de OS reduziu os sítios de ligação para água e aumentou a compatibilidade entre o amido e o PBAT.

O aumento da concentração de óleo para $1 \%$ causou aumento da PVA e do coeficiente de difusão no intervalo de 33-64\%. Garcia et al. ${ }^{[11]}$ verificaram que o aumento do teor de óleo em filmes de amido diminuiu a cristalinidade de filmes de amido o que favoreceu o processo de permeação do vapor de água já que a permeabilidade é menor nas zonas cristalinas. Na presença de TW filmes com $1 \%$ ou $0,5 \%$ de OS apresentaram valores de $\mathrm{D}^{\mathrm{w}}$ similares, indicando que a presença do surfatante pode ter aumentar a cristalinidade dos filmes com $1 \%$ de OS.

Filmes adicionados de $0,5 \%$ de TW (F3 e F4) apresentaram maiores valores de $\beta$ em comparação com os demais filmes, mas não houve variação da PVA dos filmes em função do teor de surfatante. $\mathrm{O}$ filme $\mathrm{F} 4$ apresentou o maior $\mathrm{m}_{0}$ comparado aos demais filmes adicionados de OS e/ou TW, seguido do filme F3, indicando que filmes com $0,5 \%$ de TW foram mais hidrofílicos. Filmes com $1 \%$ de TW (F5 e F6) apresentaram valores de $\mathrm{m}_{0}$ e $\beta$ similares aos filmes adicionados somente com óleo (F1 e F2). O efeito pode estar relacionado com a razão entre a porção hidrofílica e hidrofóbica do surfatante em relação às porções hidrofóbicas do filme, sendo que na condição de adição de $0,5 \%$ TW foi favorecida a sorção de água na matriz deste filmes (Figura 1).

\section{Conclusões}

Foi possível obter, por extrusão de sopro em balão, filmes biodegradáveis de amido/PBAT com alto teor de amido, com boa processabilidade, principalmente na presença de óleo. A adição de óleo de soja em filmes obtidos por blendas entre amido/PBAT conferiu maior barreira ao vapor de água. Filmes de amido/PBAT adicionados de óleo de soja foram menos hidrofílicos. A redução dos coeficientes de solubilidade e difusão indica que o óleo exerceu efeito de barreira aos vapores de água nos filmes elaborados. A incorporação de óleo aumentou as porções hidrofóbicas na matriz dos filmes, sendo este efeito responsável pelos resultados encontrados. A adição de surfatante propiciou filmes mais hidrofílicos, o efeito se deve ao aumento da água de sorção devido as porções hidrofílicas do surfatante.

\section{Agradecimentos}

Os autores agradecem a CAPES e ao CNPq pelo fomento à pesquisa.

\section{Referências Bibliográficas}

1. Zullo, R. \& Iannace, S. - Carbohydr. Polym., 77, p.376 (2009). http:// dx.doi.org/10.1016/j.carbpol.2009.01.007

2. Ren, J.; Fu, H.; Ren, T. \& Yuan, W. - Carbohydr. Polym., 77, p.576 (2009). http://dx.doi.org/10.1016/j.carbpol.2009.01.024

3. Sarazin, P.; Li, G.; Orts, W. J. \& Favis, B. D. - Polymer, 49, p.599 (2008). http://dx.doi.org/10.1016/j.polymer.2007.11.029

4. Thunwall, M.; Kuthanová, V.; Boldizar, A. \& Rigdah, L. M. - Carbohydr. Polym., 71, p.583 (2008). http://dx.doi.org/10.1016/j. carbpol.2007.07.001 
5. BASF. - "Production Information Ecoflex F BX 7011". Disponível em: <www. basf.de/ecoflex>. Acesso em: 20 jun 2012.

6. Brandelero, R. P. H.; Grossmann, M. V. E. \& Yamashita, F. - Carbohydr. Polym., 86, p.1344 (2011). http://dx.doi.org/10.1016/j. carbpol.2011.06.045

7. The, D. P.; Debeaufort, F.; Voilley, A. \& Luu, D. - Food Hydrocoll. oids, 23, p.691 (2009).

8. Pellicano, M.; Pachekoski, W. \& Agnelli, J. - Polímeros, 19, p.212 (2009). http://dx.doi.org/10.1590/S0104-14282009000300009

9. Liu, L.; Kerry, J. F. \& Kerry, J. P. - Int. J. Food Sci. Technol., 2006, 41, p.295 (2006). http://dx.doi.org/10.1111/j.1365-2621.2005.01063.x

10. Brandelero, R. P. H.; Yamashita, F. \& Grossmann, M. V. E. - Carbohydr. Polym., 82, p.1102 (2010). http://dx.doi.org/10.1016/j. carbpol.2010.06.034

11. García, M. A.; Martino, M. N. \& Zaritzky, N. E. - Food Chem. Toxicol., v. 665,6, p.941 (2000).

12. Chen, C.; Kuo, W. \& Lai, L. - Food Hydrocoll., 23, p.714 (2009). http:// dx.doi.org/10.1016/j.foodhyd.2008.06.006

13. Jensen, S.; Grosmann, M. V. E. \& Mali, S. - Braz. J. Food Technol., 12, p.97 (2009).

14. Villalobos, R.; Hernández-Muñoz,P. \& Chiralf, A. - Food Hydrocoll., 20, p.502 (2006). http://dx.doi.org/10.1016/j.foodhyd.2005.04.006

15. Rodrígues, M.; Osés, J.; Ziani, K. \& Maté, J. I. - Food Res. Int., 39, p.840 (2006).

16. Bertuzzi, M. A.; Vidaurre, E. F. C.; Armada, M. \& Gottifredi, J. C. - J. Food Eng., 80, p.977 (2007).
17. Müller, C.M.O.; Yamashita,F. \& Laurindo, J.B.-Carbohydr. Polym.,72, p.82 (2008). http://dx.doi.org/10.1016/j.carbpol.2007.07.026

18. Mali, S.; Sakanaka, L. S.; Yamashita, F. \& Grossmann, M. V. E. - Carbohydr. Polym., 60, p.283 (2005). http://dx.doi.org/10.1016/j. carbpol.2005.01.003

19. American Society for Testing And Materials - ASTM. - "E 96-95: Standard Test Methods for Water Vapor Transmission of Material”, in: Annual Book of ASTM Standards, Philadelphia, ASTM (1995).

20. Larotonda, F. D. S.; Matsui, K. N.; Sobral, P. J. A. \& Laurindo, J. B. - J. Food Eng., 71, p.394 (2005). http://dx.doi.org/10.1016/j. jfoodeng.2004.11.002

21. Martelli, S. M.; Moore, G.; Paes, S. S.; Gandolfo C. \& Laurindo, J. B. - LWT - Food Sci. Technol., 39, p.292 (2006).

22. Costa, D. L. M. - "Produção por extrusão de filmes de alto teor de amido termoplástico com poli(butileno adipato co-tereftalato) (PBAT)", Dissertação de Mestrado, Universidade Estadual de Londrina, Brasil (2008).

23. Matta Junior, M. D.; Sarmento, S. B. S.; Sarantópoulos, C. I. G. L. \& Zocchi, S. S. - Polímeros, 21, p.67 (2011).

24. Krochta, J. M. \& De Mulder-Johnston, C. - Food Technol., 51, p.61 (1997).

Enviado: 10/04/12

Reenviado: 29/06/12

Aceito: 05/07/12 